\title{
Configuration Effects on Local Transport in High-Beta LHD Plasmas
}

\author{
Hisamichi FUNABA, Kiyomasa WATANABE, Sadayoshi MURAKAMI ${ }^{1)}$, \\ Satoru SAKAKIBARA, Hiroshi YAMADA, Kazumichi NARIHARA, Ichihiro YAMADA, \\ Kenji TANAKA, Tokihiko TOKUZAWA, Masaki OSAKABE, Junichi MIYAZAWA, \\ Masayuki YOKOYAMA, Kazuo KAWAHATA and the LHD Experimental Group \\ National Institute for Fusion Science, Toki, Gifu 509-5292, Japan \\ 1) Department of Nuclear Engineering, Kyoto University, Kyoto 606-8501, Japan
}

(Received 4 September 2007 / Accepted 25 February 2008)

\begin{abstract}
To distinguish between the beta effect and configuration effect due to an increment in beta on the gradual degradation of the global confinement property in high-beta plasmas on the Large Helical Device (LHD), the local transport characteristics in the high-beta plasmas are studied by considering the change in the major radius of the magnetic flux surface with the beta value. First, the influence of the change in the magnetic configuration on the local transport is studied in low-beta plasmas, and it is confirmed that the dependence of the local transport properties on the magnetic configuration is almost the same as that proposed in the ISS04 scaling in the entire plasma region. Next, the dependence of the local transport characteristics in high-beta plasmas on the major radial position of a geometric center of the magnetic flux surface is studied in comparison with that in low-beta plasmas. The dependence of the local transport in the peripheral region is correlated more with beta itself than the magnetic configuration effect, whereas the core transport appears to be correlated more with the configuration effect.

(c) 2008 The Japan Society of Plasma Science and Nuclear Fusion Research
\end{abstract}

Keywords: high beta plasma, magnetic configuration, global confinement, local transport, international Stellarator/Heliotron scaling

DOI: $10.1585 /$ pfr.3.022

\section{Introduction}

The operation area of the Large Helical Device (LHD) has been expanded into the high-beta region, since almost $5 \%$ of the volume averaged beta $\langle\beta\rangle$ is required for heliotron type reactors. Recently, plasmas with a $\langle\beta\rangle$ value close to $5 \%$ have been obtained in LHD [1]. Although the low-order magnetic fluctuations that resonate with the rational surfaces in the peripheral region were observed in such plasmas, the increment in the amplitude of fluctuations with beta is gradual, and no disruptive increment in magnetic fluctuations was observed. When the heating power is increased, the obtained beta value also increases, and no abrupt degradation of the confinement property is observed. However, from the results of the global confinement analysis, gradual confinement degradation was observed in high-beta regimes. The purpose of this study is to clarify the causes of this gradual degradation, and investigate the confinement property in the high-beta regime corresponding to a fusion reactor.

Thus far, transport study of helical plasmas has been conducted mainly for low-beta plasmas up to now. The activity of the International Stellarator/Heliotron Scaling (ISHS) has intensively progressed. Scaling laws of the energy confinement time for helical plasmas have been

author's e-mail: funaba@lhd.nifs.ac.jp proposed by this activity (ISS95: International Stellarator Scaling 1995 [2], ISS04: International Stellarator Scaling 2004) [3]). The ISS04 scaling proposes a unified scaling law for helical plasmas that have a wide variety of magnetic configurations by introducing a renormalization factor, which is considered to originate from the magnetic configuration. According to the ISS04 scaling, although the confinement property depends on the magnetic configuration, its dependence on plasma parameters, such as beta value, collisionality, and normalized Larmor radius is considered to be similar to the gyro-reduced Bohm (GRB) type model. For LHD, the more torus-inward shifted magnetic configurations have better confinement properties in the configuration range of the magnetic axis position in vacuum $R_{\mathrm{ax}}^{\mathrm{vac}} \geq 3.60 \mathrm{~m}$, where the data of LHD are included in the International Stellarator/Heliotron Confinement Database (ISHCDB).

With respect to the transport study for the LHD plasmas, transport analysis for plasmas in the highcollisionality region near the density limit [4] and in the high-beta region [5] is performed in addition to the above ISHS activity. In such plasmas, it is seen that the dependence on the plasma parameters is different from the ISS95 or ISS04 scalings.

From the results of the local transport analysis in the 
high-beta regime in Ref. [5], transport degradation with beta increment was stronger in the peripheral region. It was found that the beta dependence of the thermal transport coefficient was similar to that of the resistive pressuregradient-driven (g-mode) transport [6]. On the other hand, in the high-beta plasmas on LHD, the magnetic flux surfaces are shifted towards the outside of torus due to the Shafranov shift. It may be possible that changes in the magnetic configuration by beta affect the dependence of the confinement property on beta in Ref. [5].

The main purpose of this study is to distinguish the causes of the gradual confinement degradation with beta from the following two effects: (1) the change in magnetic configuration by the increment in beta, and (2) the effects caused by beta other than above (1), i.e., the magnitude of beta, or the gradient of beta in the minor radial direction. The global confinement study is conducted by considering the change in the magnetic configuration by the increment in beta. Then, an analysis of the dependence of the local transport properties on magnetic configuration or the beta value is performed.

This paper consists of the following sections. In Sec. 2, the experimental setup and the method of transport analysis are described. The change in the magnetic flux surface structure due to the increment in beta and its effect on the global confinement characteristics are shown in Sec. 3. The dependence of the local transport on the magnetic configuration in the low-beta regime is investigated in Sec. 4. In Sec. 5, the relation between the local transport coefficients and the change in the geometric center position of the magnetic flux surface by the increment in beta is shown. Then, its effect on the confinement degradation in the high-beta region is discussed and a summary is provided in Sec. 6.

\section{Experimental Setup and the Method of Transport Analysis}

LHD is a heliotron-type device with a poloidal period number as $l=2$ and a toroidal period number as $m=10$. The major radius position of the magnetic axis in vacuum, $R_{\mathrm{ax}}^{\mathrm{vac}}=3.5-4.1 \mathrm{~m}$, and the average minor radius is approximately $0.6 \mathrm{~m}$. The magnetic field strength at the toroidally averaged magnetic axis in vacuum $B_{0}^{\text {ax }}$ is up to approximately $3 \mathrm{~T}$. High-beta $(\beta)$ plasmas are produced by the high-power neutral beam injection (NBI) heating of more than $10 \mathrm{MW}$ and in the condition of relatively low magnetic field strength. The discharges in the low magnetic field are initiated by NBI heating alone.

The plasma stored energy obtained from diamagnetic measurement $W_{\mathrm{p}}^{\mathrm{dia}}$ is used for the evaluation of the global energy confinement property. The value of the volumeaverage beta $\left\langle\beta^{\text {dia }}\right\rangle$ is also derived from the diamagnetic measurement [7]. The profiles of the electron temperature $T_{\mathrm{e}}$ are measured by Thomson scattering with YAG lasers. The $T_{\mathrm{e}}$ profiles in the real coordinate are converted into the profile in the magnetic coordinate using the optimum mapping data, which is selected from the equilibrium database based on the symmetry of the profile in the torus-inside and -outside regions of the magnetic axis. Profiles of the electron density $n_{\mathrm{e}}$ are estimated from Abel inversion of the data measured using a far infrared (FIR) laser interferometer. In this inversion, the same mapping data of the magnetic flux surface are used in the evaluation of the $T_{\mathrm{e}}$ profiles.

The power deposition profiles of NBI are calculated by a three-dimensional Monte Carlo simulation code [8]. In this calculation, particles that go outside the outermost magnetic flux surface during some rotations in the poloidal direction are considered as the source of heat loss by direct orbit loss mechanism. These NBI deposition profiles, used in the local transport analysis include the broadening from the birth profiles of the high-energy ions by NBI due to the finite orbit effect. Here, the magnitude of the ionized power of NBI in the calculation is set to be equal to the experimentally estimated NBI absorbed power, which is the port-through power minus the shine-through power, and contains approximately $10 \%$ error [9].

The thermal transport coefficients of electrons $\chi_{\mathrm{e}}^{\exp }$ and ions $\chi_{\mathrm{i}}^{\text {exp }}$ are evaluated using a one-dimensional transport code for helical plasmas, PROCTR [10]. Here, we assume that $T_{\mathrm{i}}=T_{\mathrm{e}}$ and $n_{\mathrm{i}}=f n_{\mathrm{e}}$, where $f$ is determined by the following relation so that the kinetic energy becomes equal to the measured stored energy:

$$
W_{\mathrm{p}}^{\mathrm{dia}}=\int \frac{3}{2}(1+f) n_{\mathrm{e}} T_{\mathrm{e}} \mathrm{d} V,
$$

where the integral is the volume integral. In this paper, the local transport is analyzed based on the measurements of $T_{\mathrm{e}}$ and $n_{\mathrm{e}}$. By assuming that $T_{\mathrm{i}}=T_{\mathrm{e}}$, the equipartition power between electrons and ions $P_{\mathrm{ei}}=0$ is assumed. Therefore, the behavior of the effective transport coefficients, which are evaluated by

$$
\chi^{\mathrm{eff}}=\left(\chi_{\mathrm{e}}^{\mathrm{exp}}+f \chi_{\mathrm{i}}^{\exp }\right) /(1+f)
$$

is studied.

The parameter region of the high-beta plasmas analyzed in this study is shown in Fig. 1 . The ordinate denotes $T_{\mathrm{e}}(0) / B_{0}^{\text {ave } 2}$, where $T_{\mathrm{e}}(0)$ is the central electron temperature, and $B_{0}^{\text {ave }}$ is the volume-averaged magnetic field strength in vacuum. The abscissa denotes the central electron density $n_{\mathrm{e}}(0)$. The dotted curves in the figure denote the contour of the central beta value, $\beta_{0}$. The symbols are grouped according to the magnetic field strength at the magnetic axis in vacuum $B_{0}^{\text {ax }}$.

The parameters of the magnetic configuration are as follows. The major radius position of the magnetic axis in vacuum $R_{\mathrm{ax}}^{\mathrm{vac}}=3.60 \mathrm{~m}$, the parameter $B_{\mathrm{q}}$, which represents the canceling ratio of the quadrupole component of the helical magnetic field, is $100 \%$. $B_{\mathrm{q}}$ is related with the ellipticity of the shape of the poloidal cross section, which 


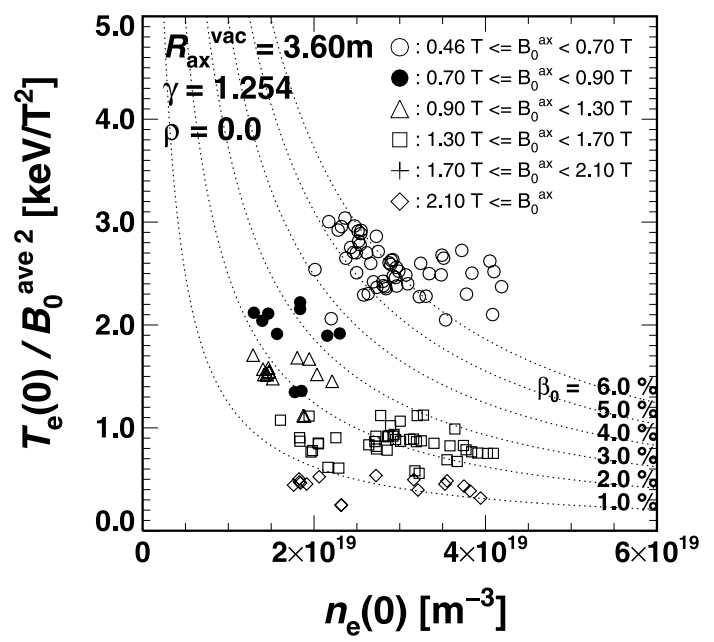

Fig. 1 Parameter range of the experimental data. The relation between $T_{\mathrm{e}}(0) / B_{0}^{\text {ave } 2}$ and $n_{\mathrm{e}}(0)$ is shown. $T_{\mathrm{e}}(0)$ is the central electron temperature and $B_{0}^{\text {ave }}$ is the average magnetic field strength in vacuum. $n_{\mathrm{e}}(0)$ is the central electron density. The dotted curves denote the contour of the central beta value $\beta_{0}$.

is averaged in the toroidal direction. $B_{\mathrm{q}}=100 \%$ corresponds to almost unity ellipticity. The plasma aspect ratio $A_{\mathrm{p}}$, on which the achieved maximum beta value strongly depends in LHD [7], is 5.8. The maximum $\left\langle\beta^{\text {dia }}\right\rangle$ in the plasmas with $A_{\mathrm{p}}=5.8$ is $3.2 \%$. These $B_{\mathrm{q}}$ and $A_{\mathrm{p}}$ values correspond to the standard in LHD experiments. The condition of $A_{\mathrm{p}}=5.8$ is expressed by $\gamma=1.254$ in the figures, where $\gamma$ is the pitch parameter of the helical coils. All plasmas are heated by NBI only. The data are limited in the parameter region of $1.5 \times 10^{19} \leq \bar{n}_{\mathrm{e}} \leq 4.3 \times 10^{19} \mathrm{~m}^{-3}$, $I_{\mathrm{p}} / B_{0} \leq 30 \mathrm{kA} / \mathrm{T}, W_{\mathrm{b}} / W_{\mathrm{p}}^{\mathrm{kin}} \leq 0.5$, where $\bar{n}_{\mathrm{e}}$ is the line averaged electron density, $I_{\mathrm{p}}$ is the plasma current, $W_{\mathrm{b}}$ is the calculated energy of the NBI beam component, and $W_{\mathrm{p}}^{\mathrm{kin}}$ is the estimated plasma stored energy based on the profiles of electron temperature and density.

\section{The Change in the Magnetic Flux Surface Structure by the Increment in Beta and its Effect on the Global Confinement Characteristics}

The ISHS activity has been investigating the global confinement property of several stellarators and heliotrons mainly for the low-beta plasmas. The energy confinement time by the ISS04 scaling $\tau_{\mathrm{E}}^{\mathrm{ISS} 04}$ is expressed as follows:

$$
\tau_{\mathrm{E}}^{\mathrm{ISS} 04}=0.134 \cdot a^{2.28} R^{0.64} P^{-0.61} \bar{n}_{\mathrm{e}}^{0.54} B^{0.84} t_{2 / 3}^{0.41},
$$

where $a, R, P, \bar{n}_{\mathrm{e}}, B$, and $t_{2 / 3}$ are the minor radius, major radius, absorbed power, line average electron density, volume averaged strength of the magnetic field, and rotational transform $t=\iota / 2 \pi$ at the normalized average minor radius $\rho=2 / 3$, respectively. This $\rho$ is defined as $\rho=\left(\Phi / \Phi_{\mathrm{a}}\right)^{1 / 2}$,
Table 1 Relation among the positions of the magnetic axis in vacuum $R_{\mathrm{ax}}^{\mathrm{vac}}$ the geometric center of the $\rho=2 / 3$ magnetic surface in vacuum $R_{\mathrm{geo}}^{\mathrm{vac}}(2 / 3)$, and $f_{\text {ren }}^{\mathrm{ISS} 04}$ in the ISS04 scaling for three magnetic configurations on LHD [3].

\begin{tabular}{|c|c|c|c|}
\hline Device & $R_{\mathrm{ax}}^{\mathrm{vac}}[\mathrm{m}]$ & $R_{\mathrm{geo}}^{\mathrm{vac}}(2 / 3)[\mathrm{m}]$ & $f_{\mathrm{ren}}^{\mathrm{ISS} 04}$ \\
\hline LHD & 3.60 & 3.643 & $0.93 \pm 0.15$ \\
\hline LHD & 3.75 & 3.740 & $0.67 \pm 0.06$ \\
\hline LHD & 3.90 & 3.849 & $0.48 \pm 0.05$ \\
\hline
\end{tabular}

where $\Phi$ is the toroidal magnetic flux, and the subscript "a" indicates the value at the edge. For the finite $\beta$ case, the $\rho=1$ surface is defined as a surface that includes the $\rho=1$ point of the vacuum magnetic flux surface at the torus-outside of the midplane. In the ISS04 scaling, the geometrical parameters $a, R, B$, and $t$ which were evaluated in vacuum were used. The absorbed power of NBI, which is evaluated as described in the previous section, is used for $P$ in this paper.

The advance from the previous ISS95 scaling to ISS04 is that the strong effect that depends on the devices or the magnetic configurations is included, although the dependence on the plasma parameters is similar. This effect is expressed as the renormalization factor $f_{\text {ren }}$ which represents the dependence on the device or the magnetic configuration. Using this $f_{\text {ren }}$, the ISS04 scaling value, which should be compared with the experimental energy confinement time $\tau_{\mathrm{E}}^{\text {exp }}$ is expressed as $f_{\text {ren }} \tau_{\mathrm{E}}^{\mathrm{ISS} 04}$. In the case of LHD, $f_{\text {ren }}$ strongly depends on the position of the magnetic axis in vacuum $R_{\mathrm{ax}}^{\mathrm{vac}}$ and the shape of the outermost magnetic flux surface. In Ref. [3], the dependence of $f_{\text {ren }}$ on $R_{\mathrm{ax}}^{\mathrm{vac}}$ is analyzed in detail, and the derived $f_{\text {ren }}$ values are

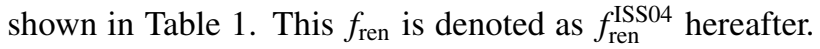

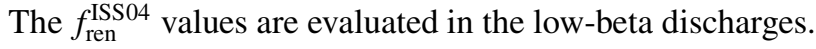
For LHD, the beta values of the plasmas are in the range of $\left\langle\beta^{\text {dia }}\right\rangle \leq 1.5 \%$.

The results of the high-beta experiments suggest that the global confinement time is degraded as the beta value increases on LHD. The dependence of the global confinement property on $\left\langle\beta^{\text {dia }}\right\rangle$ is shown in Fig. 2. The ordinate represents the ratio of $\tau_{\mathrm{E}}^{\mathrm{exp}} /\left(f_{\mathrm{ren}}^{\mathrm{ISS} 04} \tau_{\mathrm{E}}^{\mathrm{ISS} 04}\right)$. The value of $f_{\mathrm{ren}}^{\mathrm{ISS} 04}$ in the case of $R_{\mathrm{ax}}^{\mathrm{vac}}=3.60 \mathrm{~m}$ is used, since all the data in Fig. 2 are obtained in the condition of $R_{\mathrm{ax}}^{\mathrm{vac}}=3.60 \mathrm{~m}$. The symbols are grouped according to the collisionality $v_{\mathrm{b}}^{*}$ which is normalized by $v_{\mathrm{b}}=\epsilon_{\mathrm{t}}^{3 / 2} \cdot v_{\text {the }} t / R$, where $\epsilon_{\mathrm{t}}$ is the inverse aspect ratio, and $v_{\text {the }}$ is the thermal electron velocity.

The symbols used to indicate the magnetic field strength, major radius, effective minor radius, and rotational transform hereafter are listed with detailed definitions in Table 2 . The major radius $R_{\text {geo }}^{\mathrm{vac}}(1)$ denotes the geometric center position of the $\rho=1$ magnetic flux surface in vacuum. The minor radius $a_{\mathrm{eff}}^{\mathrm{vac}}$ is derived from the volume 


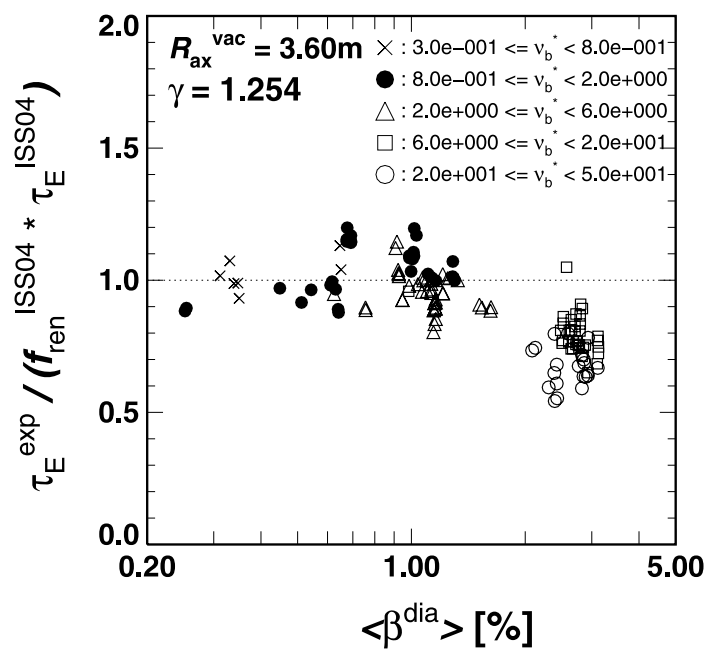

Fig. $2\left\langle\beta^{\text {dia }}\right\rangle$ dependence of $\tau_{\mathrm{E}}^{\exp } /\left(f_{\text {ren }}^{\mathrm{ISS} 04} \tau_{\mathrm{E}}^{\mathrm{ISS} 04}\right)$, where $\tau_{\mathrm{E}}^{\exp }$ is the experimentally derived energy confinement time and $\tau_{\mathrm{E}}^{\mathrm{ISS} 04}$ is the energy confinement time evaluated by ISS04 scaling. $f_{\text {ren }}^{\mathrm{ISS} 04}$ is the renormalization factor in ISS04.

of the $\rho=1$ magnetic flux surface in vacuum. In Fig. 2, $R_{\mathrm{geo}}^{\mathrm{vac}}(1), a_{\mathrm{eff}}^{\mathrm{vac}}, B_{0}^{\text {ave }}$, and $t_{2 / 3}^{\mathrm{vac}}$, where the superscript "vac" indicates that these values are evaluated in vacuum, are used as $R, a, B$, and $t_{2 / 3}$ in Eq. (3). It should be noted that these definitions are the same as those in Ref. [3]. When $\left\langle\beta^{\text {dia }}\right\rangle$ increases from 1 to $3.2 \%$, the confinement property degrades by about $30 \%$ on average. The quantity of MHD equilibrium changes with the increment in the beta value. The relationships between $\left\langle\beta^{\text {dia }}\right\rangle$ and the geometric center position of the $\rho=1$ magnetic flux surface $R_{\text {geo }}(1)$ and the plasma minor radius $a^{\mathrm{Wp} 99}$ [11] are shown in Fig. 3 (a) and (b), respectively. The parameter $a^{\mathrm{Wp} 99}$ is evaluated from the volume $V^{\mathrm{Wp} 99}$ which includes $99 \%$ of the integrated electron pressure. The electron pressure is derived as the product of the electron temperature and density. It is found that $R_{\text {geo }}(1)$ moves torus-outward with the increment in $\left\langle\beta^{\text {dia }}\right\rangle$. The minor radius $a^{\mathrm{Wp} 99}$ gradually increases with the beta value, where the change due to beta is about $10 \%$.

Figure 4 shows the relation between $\left\langle\beta^{\text {dia }}\right\rangle$ and the geometric center position of the $\rho=2 / 3$ magnetic flux surface $R_{\text {geo }}(2 / 3)$, which is used as a parameter that represents the property of a magnetic configuration. $R_{\text {geo }}(2 / 3)$ changes steeply around $\left\langle\beta^{\text {dia }}\right\rangle \sim 3 \%$, and increases to about $3.8 \mathrm{~m}$, indicating that the fractional change due to beta is about $5 \%$. Figure 5 shows the dependence of $t_{2 / 3}$ on $\left\langle\beta^{\text {dia }}\right\rangle$. The change in $t_{2 / 3}$ due to beta is about $10 \%$. Therefore, the parameters that represent the structure of the magnetic configuration vary with the change in MHD equilibrium due to the increment in beta.

The dependence of the global energy confinement time normalized by ISS0 4 on $\left\langle\beta^{\text {dia }}\right\rangle$ is rewritten in Fig. 6 using $R_{\text {geo }}(1), a^{\mathrm{Wp} 99}$, and $t_{2 / 3}$ of the finite beta equilibrium, as shown in Figs. 3 and 5. The ratio $\tau_{\mathrm{E}}^{\exp } /\left(f_{\text {ren }} \tau_{\mathrm{E}}^{\operatorname{ISS} 04(\beta)}\right)$ seems to be slightly larger than unity in the low-beta
Table 2 Definitions of symbols of some parameters that are used to derive the scaling values. The "equilibrium" column denotes that the parameter is derived for the vacuum case or the finite beta case.

\begin{tabular}{|c|c|c|c|}
\hline $\begin{array}{l}\text { parame- } \\
\text { ter }\end{array}$ & symbol & definition & $\begin{array}{l}\text { equilib- } \\
\text { rium }\end{array}$ \\
\hline \multirow[b]{2}{*}{$\begin{array}{l}\text { magnetic } \\
\text { field } \\
\text { strength }\end{array}$} & $B_{0}^{\mathrm{ax}}$ & at the magnetic axis & vacuum \\
\hline & $B_{0}^{\text {ave }}$ & $\begin{array}{l}\text { volume averaged, } \\
\Phi_{\mathrm{a}}=\pi a_{\mathrm{eff}}^{\mathrm{vac} 2} B_{0}^{\text {ave }}\end{array}$ & vacuum \\
\hline \multirow{4}{*}{$\begin{array}{l}\text { major } \\
\text { radius }\end{array}$} & $R_{\mathrm{ax}}^{\mathrm{vac}}$ & magnetic axis & vacuum \\
\hline & $R_{\mathrm{geo}}^{\mathrm{vac}}(1)$ & $\begin{array}{l}\text { geometric center of } \\
\text { the } \rho=1 \text { magnetic } \\
\text { flux surface }\end{array}$ & vacuum \\
\hline & $R_{\mathrm{geo}}(1)$ & $\begin{array}{l}\text { geometric center of } \\
\text { the } \rho=1 \text { magnetic } \\
\text { flux surface }\end{array}$ & $\begin{array}{l}\text { finite } \\
\text { beta }\end{array}$ \\
\hline & $R_{\text {geo }}(2 / 3)$ & $\begin{array}{l}\text { geometric center of } \\
\text { the } \rho=2 / 3 \text { magnetic } \\
\text { flux surface }\end{array}$ & $\begin{array}{l}\text { finite } \\
\text { beta }\end{array}$ \\
\hline \multirow[t]{2}{*}{$\begin{array}{l}\text { effective } \\
\text { minor } \\
\text { radius }\end{array}$} & $a_{\mathrm{eff}}^{\mathrm{vac}}$ & $\begin{array}{l}\text { derived from the vol- } \\
\text { ume in vacuum. } a_{\mathrm{eff}}^{\mathrm{vac}}= \\
\left\{V^{\mathrm{vac}} /\left(2 \pi^{2} R_{\mathrm{geo}}^{\mathrm{vac}}(1)\right)\right\}^{1 / 2} \text {, } \\
\text { where } V^{\mathrm{vac}} \text { is the vol- } \\
\text { ume enclosed by the } \\
\text { the vacuum magnetic } \\
\text { flux surface of } \rho=1 \text {. }\end{array}$ & vacuum \\
\hline & $a^{\mathrm{Wp} 99}$ & $\begin{array}{l}\text { derived from the } \\
\text { volume }\left(V^{\mathrm{Wp} 99}\right) \text { and } \\
\text { the major radius of } \\
\text { the geometric center } \\
\text { position }\left(R_{\mathrm{geo}}^{\mathrm{Wp} 99}\right) \text { of the } \\
\text { magnetic flux surface } \\
\text { which includes } 99 \% \\
\text { of the integrated elec- } \\
\text { tron pressure. } a^{\mathrm{Wp} 99}= \\
\left\{V^{\mathrm{Wp} 99} /\left(2 \pi^{2} R_{\mathrm{geo}}^{\mathrm{Wp} 99}\right)\right\}^{1 / 2} \text {. }\end{array}$ & $\begin{array}{l}\text { finite } \\
\text { beta }\end{array}$ \\
\hline \multirow[t]{2}{*}{ rotational } & $t_{2 / 3}^{\mathrm{vac}}$ & $t$ at $\rho=2 / 3$ & vacuum \\
\hline & $t_{2 / 3}$ & $t$ at $\rho=2 / 3$ & $\begin{array}{l}\text { finite } \\
\text { beta }\end{array}$ \\
\hline
\end{tabular}

regime, mainly because $a^{\mathrm{Wp} 99}$ in the low-beta region is smaller than $a_{\mathrm{eff}}^{\mathrm{vac}}\left(a^{\mathrm{Wp} 99} \simeq 0.61 \mathrm{~m}\right.$ at $\left\langle\beta^{\mathrm{dia}}\right\rangle \sim 0.5 \%$ and $\left.a_{\mathrm{eff}}^{\mathrm{vac}}=0.64 \mathrm{~m}\right)$. Here, $\tau_{\mathrm{E}}^{\mathrm{ISS} 04(\beta)}$ denotes the scaling value, which is estimated based on the geometrical parameters in finite beta. As shown in Fig. 6, the gradual confinement degradation in the high-beta region is observed even when the finite beta parameters are used.

In the ISS04 scaling, the renormalization factor $f_{\text {ren }}^{\text {ISS04 }}$ is included to represent the dependence of devices or magnetic configurations. The value of $f_{\text {ren }}^{\text {ISSO4 }}$ for LHD depends on the vertical magnetic field produced by the poloidal 
(a)

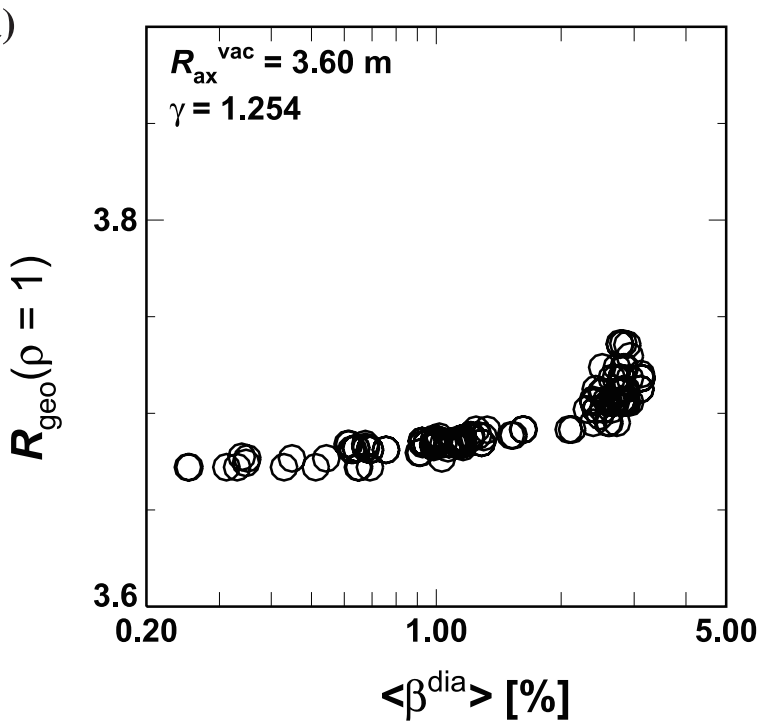

(b)

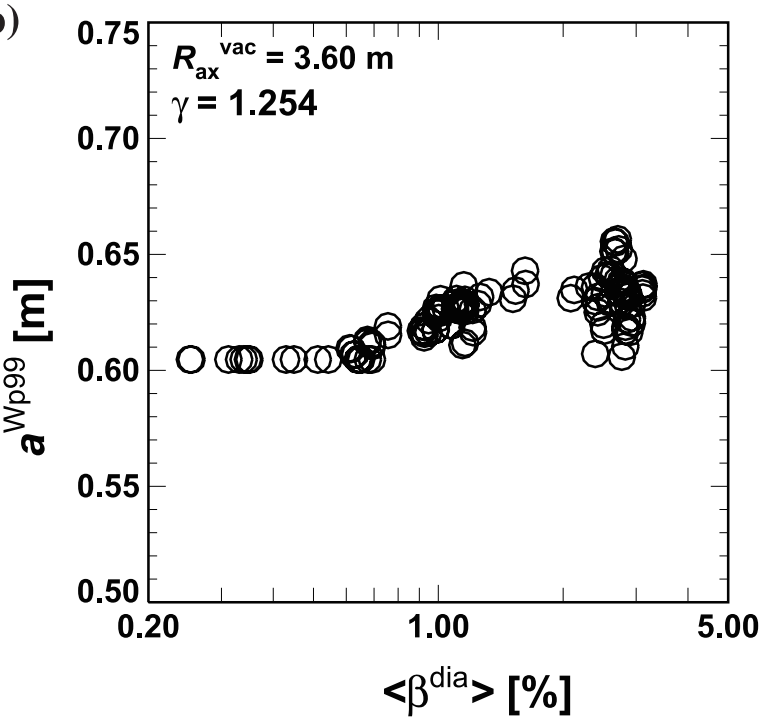

Fig. 3 (a) $\left\langle\beta^{\text {dia }}\right\rangle$ dependence of the major radius of the geometric center of the outermost magnetic flux surface $R_{\mathrm{geo}}(1)$. (b) $\left\langle\beta^{\text {dia }}\right\rangle$ dependence of the plasma minor radius $a^{\mathrm{Wp}_{\mathrm{p} 9} \text {, }}$ evaluated based on the volume involving $99 \%$ of the integrated electron pressure.

coils and the quadrupole component of the helical field. With respect to the change in the magnetic field by the finite beta effect, the effect of the vertical field by the PfirshSchluter current is stronger than that of the quadrupole component. The major radial position of the magnetic axis or the geometric center of the magnetic flux surface is shifted by the vertical magnetic field. Therefore, it is assumed that $f_{\text {ren }}$ is expressed as a function of the geometric center of the magnetic flux surface at $\rho=2 / 3$, denoted by $R_{\text {geo }}(2 / 3)$, which is considered to be a parameter representing a magnetic configuration in this paper. The relationship between $R_{\text {geo }}(2 / 3)$ and $f_{\text {ren }}^{\mathrm{ISS} 04}$ is shown in Fig. 7. The dotted curve shows an interpolation by a quadratic function of $R_{\text {geo }}(2 / 3)$. To evaluate the global scaling value, which

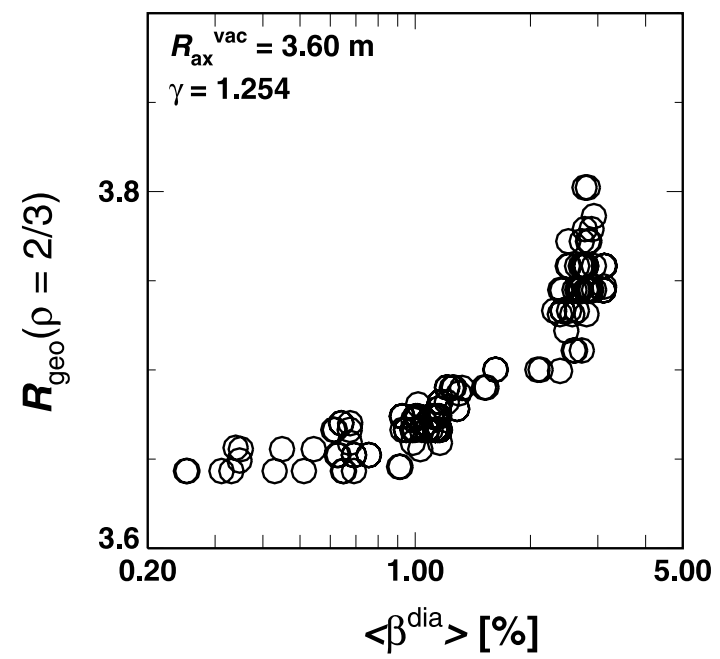

Fig. 4 Dependence of the radial position of the center of the $\rho=$ $2 / 3$ magnetic flux surface $R_{\mathrm{geo}}(2 / 3)$ on $\left\langle\beta^{\mathrm{dia}}\right\rangle$.

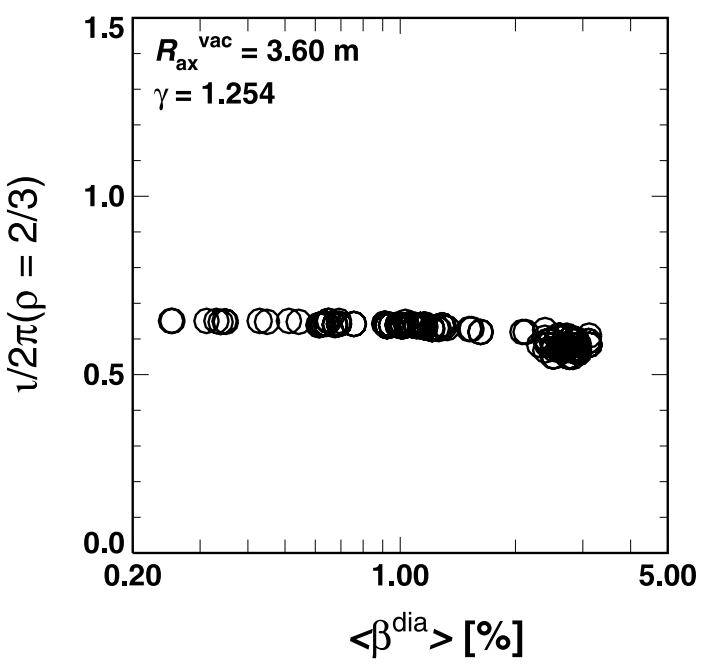

Fig. 5 Dependence of $t$ at the $\rho=2 / 3$ magnetic flux surface on $\left\langle\beta^{\text {dia }}\right\rangle$.

includes the effects of the change in the magnetic configuration, an interpolated value of the renormalization factor $f_{\text {ren }}^{\text {int }}$ is used.

The dependence of the global energy confinement time normalized by ISSO4 on $\left\langle\beta^{\mathrm{dia}}\right\rangle$ is rewritten in Fig. 8 using $f_{\text {ren }}^{\text {int }}$ instead of $f_{\text {ren }}^{\mathrm{ISS} 04}$ at $R_{\mathrm{ax}}^{\mathrm{vac}}=3.60 \mathrm{~m}$ in addition to the finite beta effect as shown in Fig. 6. The degradation due to the increment in beta seems to disappear. In contrast to Fig. 6 where only the parameters in the finite beta case are included in the scaling, the interpolation of $f_{\text {ren }}^{\text {ISS04 }}$ needs to be considered to compensate for the apparent degradation. This suggests that the global confinement degradation may seemingly be caused by the change in the magnetic configuration by finite beta. However, it is not obvious that this result of the global confinement coincides with the property of the local transport. The local transport 


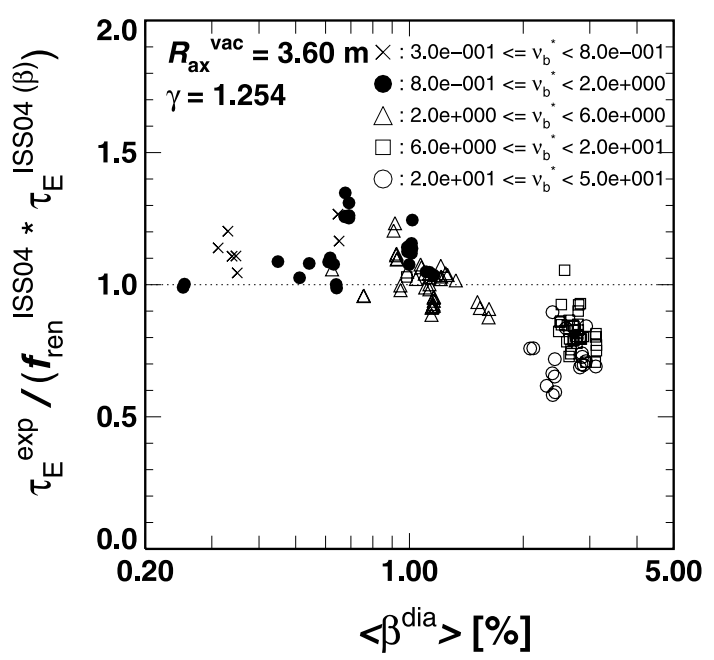

Fig. $6\left\langle\beta^{\text {dia }}\right\rangle$ dependence of $\tau_{\mathrm{E}}^{\exp } /\left(f_{\text {ren }}^{\mathrm{ISS} 04} \tau_{\mathrm{E}}^{\mathrm{ISS} 04(\beta)}\right)$. Here, the parameters in the finite beta case are used for evaluation of $\tau_{\mathrm{E}}^{\mathrm{ISS} 04(\beta)}$.

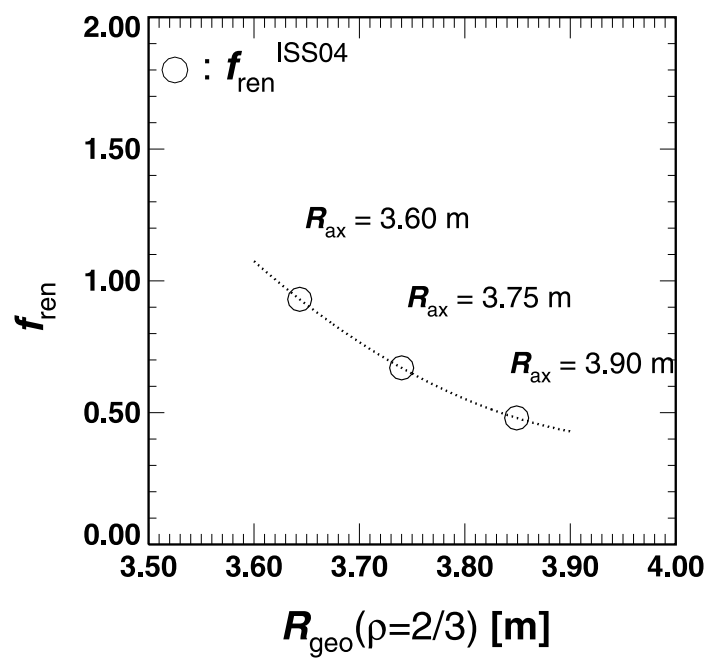

Fig. 7 Relation between $f_{\text {ren }}$ and $R_{\text {geo }}(2 / 3) . f_{\text {ren }}$ is interpolated by a quadratic function of $R_{\text {geo }}(2 / 3)$ (dotted curve).

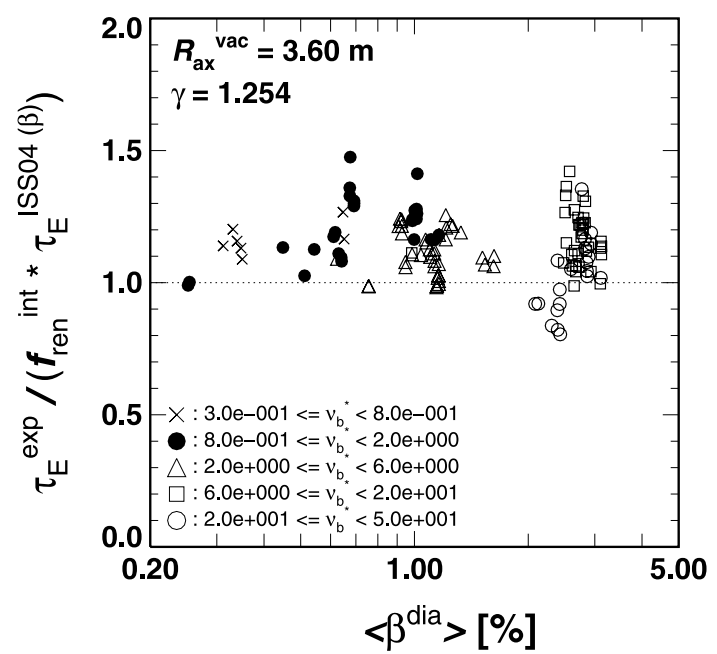

Fig. $8\left\langle\beta^{\text {dia }}\right\rangle$ dependence of $\tau_{\mathrm{E}}^{\exp } /\left(f_{\mathrm{ren}}^{\text {int }} \tau_{\mathrm{E}}^{\mathrm{ISS} 04}\right)$. where $f_{\text {ren }}^{\text {int }}$ is the interpolated renormalization factor for $R_{\text {geo }}(2 / 3)$. is analyzed in the following sections.

\section{Dependence of the Local Transport on the Magnetic Configuration in the Low-Beta Regime}

In this section, the dependence of local transport on magnetic configurations is investigated in the low-beta region to clarify the causes of the degradation of the local transport property in the high beta region.

Using the non-dimensional parameters, such as the normalized Larmor radius $\rho^{*}$, the normalized collisionality $v_{\mathrm{b}}^{*}, \beta, A_{\mathrm{p}}$, and $t_{2 / 3}, \tau_{\mathrm{E}}^{\mathrm{ISS} 04}$ is expressed as follows:

$$
\tau_{\mathrm{E}}^{\mathrm{ISS} 04}=C_{\tau} \cdot \tau_{\mathrm{Bohm}} \cdot \rho^{*-0.79} \beta^{-0.19} v_{\mathrm{b}}^{* 0.00} A_{\mathrm{p}}^{0.07} \boldsymbol{t}_{2 / 3}^{1.06},
$$

where $\tau_{\text {Bohm }}$ is the global confinement time based on the Bohm-type scaling and $C_{\tau}$ is a constant. Then, a modeled transport coefficient $\chi^{\text {ISS04 }}$ which has the same nondimensional parameter dependence as ISS04, is introduced for use as a reference for the local transport coefficient,

$$
\chi^{\mathrm{ISS} 04}=C_{\chi} \cdot \chi^{\mathrm{Bohm}} \cdot \rho^{* 0.79} \beta^{0.19} v_{\mathrm{b}}^{* 0.00} A_{\mathrm{p}}^{-0.07} \boldsymbol{t}_{2 / 3}^{-1.06} .
$$

By comparing $\chi^{\text {eff }} / \chi^{\mathrm{ISS} 04}$ in different magnetic configurations, the dependence of the local transport on the effects except the above non-dimensional parameters can be evaluated. Here, for using $\chi^{\text {eff }} / \chi^{\mathrm{ISS} 04}$ of the $R_{\mathrm{ax}}^{\mathrm{vac}}=3.60 \mathrm{~m}$ configuration as the reference in the comparison among the different configurations, $C_{\chi}$ is determined such that the average of the values of $\chi^{\text {eff }} / \chi^{\text {ISS04 }}$ becomes unity in the lowbeta region $\left(\left\langle\beta^{\mathrm{dia}}\right\rangle<1 \%\right)$ in the data set of $R_{\mathrm{ax}}^{\mathrm{vac}}=3.60 \mathrm{~m}$. The coefficient $C_{\chi}$ has different values at different $\rho$ positions (Table 3 ). Therefore, the magnitude of $\chi^{\text {eff }} / \chi^{\text {ISS04 }}$ represents the ratio of the normalized transport coefficients to the value in the configuration of $R_{\mathrm{ax}}^{\mathrm{vac}}=3.6 \mathrm{~m}$ at each minor radial position.

In Fig. $9, \chi^{\mathrm{eff}} / \chi^{\mathrm{ISS} 04}$ at the peripheral region of $\rho=0.9$ in the low-beta regime $\left(\left\langle\beta^{\text {dia }}\right\rangle \leq 1 \%\right)$ are shown for three different $R_{\mathrm{ax}}^{\mathrm{vac}}$ positions ((a) $R_{\mathrm{ax}}^{\mathrm{vac}}=3.60 \mathrm{~m}$, (b) $R_{\mathrm{ax}}^{\mathrm{vac}}=$ $3.75 \mathrm{~m}$, and (c) $R_{\mathrm{ax}}^{\mathrm{vac}}=3.90 \mathrm{~m}$ ). The abscissa is the local beta value in these figures. Although the data are scattered, $\chi^{\text {eff }} / \chi^{\text {ISS04 }}$ increases as $R_{\mathrm{ax}}^{\mathrm{vac}}$ moves torus-outward. This tendency of the local transport coefficients seems to agree qualitatively with the global confinement property. The broken line in each figure, which denotes the average

Table 3 The normalized average minor radius $\rho$, and the coefficient in the $\chi^{\mathrm{ISS} 04}$ expression $C_{\chi}$.

\begin{tabular}{|c|c|}
\hline$\rho$ & $C_{\chi}$ \\
\hline 0.5 & 0.104 \\
\hline 0.7 & 0.133 \\
\hline 0.9 & 0.294 \\
\hline
\end{tabular}


(a)

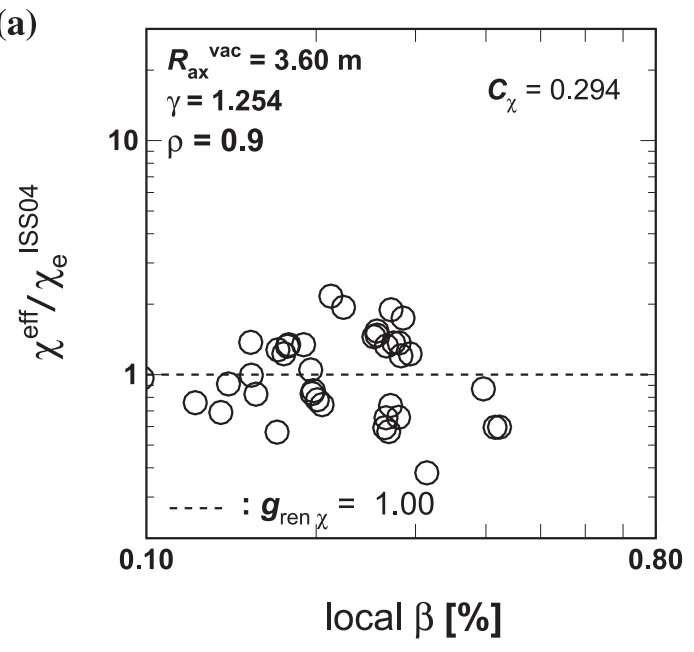

(b)

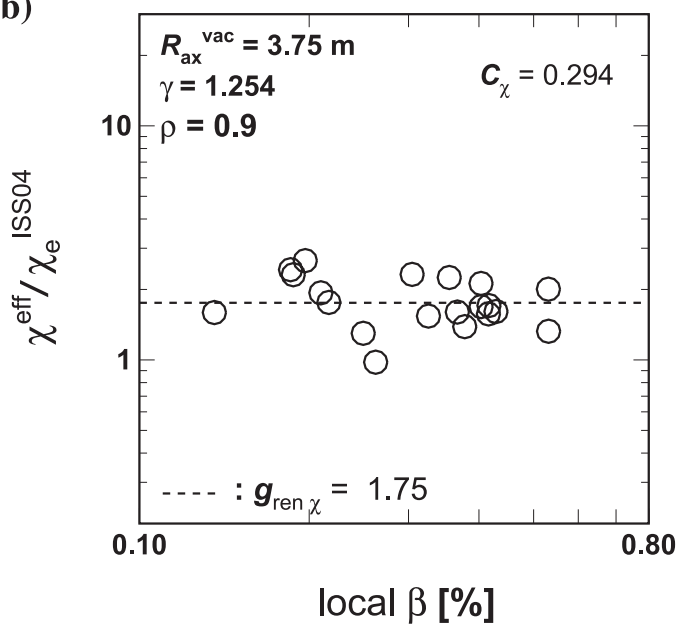

(c)

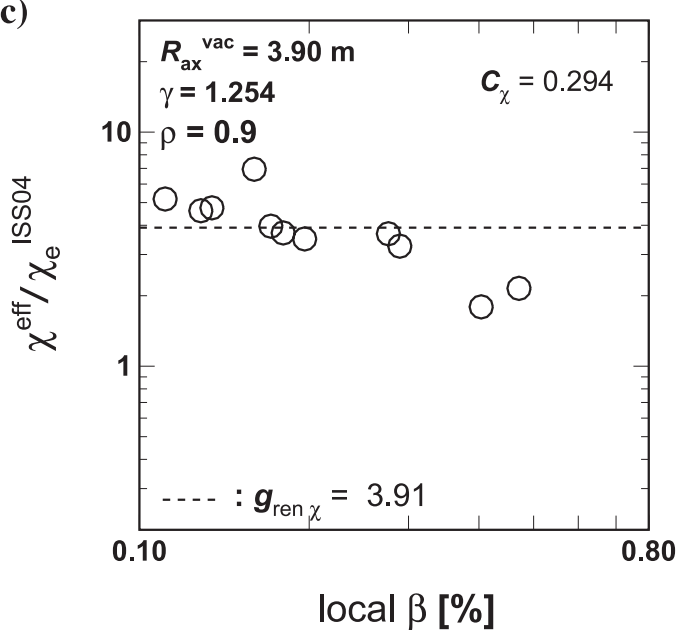

Fig. 9 Dependence of $\chi^{\text {eff }} / \chi^{\mathrm{ISS} 04}$ on local $\beta$ at $\rho=0.9$. (a) $R_{\mathrm{ax}}^{\mathrm{vac}}=$ $3.60 \mathrm{~m}$, (b) $R_{\mathrm{ax}}^{\mathrm{vac}}=3.75 \mathrm{~m}$, and (c) $R_{\mathrm{ax}}^{\mathrm{vac}}=3.90 \mathrm{~m}$.

value of the experimental results, is defined as the renormalization factor for the local transport coefficients $g_{\text {ren } \chi}$ at this local position. The parameter $g_{\text {ren } \chi}$ represents the change in the local transport coefficients in different magnetic configurations, as $f_{\text {ren }}$ denotes the change in the confinement time. The behavior of $\chi^{\text {eff }} / \chi^{\mathrm{ISS} 04}$ is also investi-

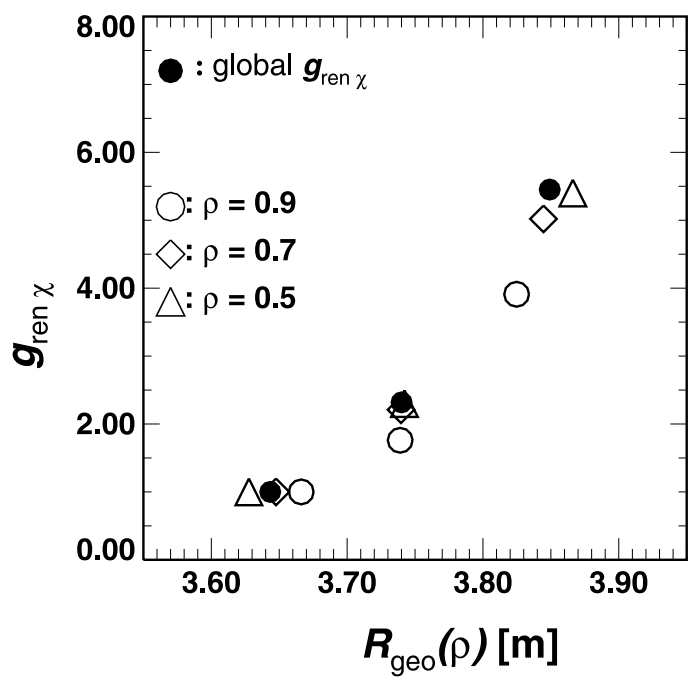

Fig. 10 Dependence of $g_{\text {ren } \chi}$ on the geometric center position of the vacuum magnetic flux surface $R_{\text {geo }}(\rho)$.

gated at $\rho=0.7$ and 0.5 . The more $R_{\mathrm{ax}}^{\mathrm{vac}}$ moves outward, the more the value of $\chi^{\text {eff }} / \chi^{\text {ISS04 }}$ increases at both local positions. This characteristic is the same as that in the peripheral region shown in Fig. 9.

The factor $g_{\text {ren } \chi}$ is derived for various magnetic configurations and radial positions. The relation between $g_{\mathrm{ren} \chi}$ and the geometric center position of each magnetic flux surface $R_{\text {geo }}(\rho)$ in vacuum is shown in Fig. 10. The symbols $\bigcirc, \diamond$ and $\triangle$ correspond to the values at $\rho=0.9,0.7$ and 0.5 , respectively. The data in $R_{\text {geo }}(\rho)=3.6-3.7 \mathrm{~m}$, $R_{\text {geo }}(\rho)=3.7-3.8 \mathrm{~m}$, and $R_{\text {geo }}(\rho)=3.8-3.9 \mathrm{~m}$ are for the configurations of $R_{\mathrm{ax}}^{\mathrm{vac}}=3.60 \mathrm{~m}, R_{\mathrm{ax}}^{\mathrm{vac}}=3.75 \mathrm{~m}$, and $R_{\mathrm{ax}}^{\mathrm{vac}}=3.90 \mathrm{~m}$, respectively. As the geometric center of the magnetic flux surface is shifted torus-outward, $g_{\mathrm{ren} \chi}$ increases at all minor radial positions.

The closed circles ( $)$ in Fig. 10 represent the renormalization factor for transport coefficients $g_{\mathrm{ren} \gamma}^{\text {ISS04 }}$, which is derived from ISS04. $g_{\mathrm{ren} x}^{\mathrm{ISS} 04}$ is related with $f_{\mathrm{ren}}^{\mathrm{ISS} 04}$, and it is compared with the renormalization factor for the local transport coefficients $g_{\mathrm{ren} \chi}$. The parameter $g_{\mathrm{ren} \chi}^{\mathrm{ISS} 04}$ is derived as follows. By assuming a spatially uniform thermal transport coefficient $\langle\chi\rangle$, the relation between the energy confinement time $\tau_{\mathrm{E}}$ and $\langle\chi\rangle$ is expressed as $\tau_{\mathrm{E}} \propto a^{2} /\langle\chi\rangle$ with the minor radius $a$. When a global scaling law is expressed as

$$
\tau_{\mathrm{E}}=f_{\mathrm{ren}} n^{\alpha_{n}} P^{\alpha_{P}} B^{\alpha_{B}} a^{\alpha_{a}} R^{\alpha_{R}},
$$

where $n$ is the density and $\alpha_{n}, \alpha_{P}, \alpha_{B}, \alpha_{a}$, and $\alpha_{R}$ are the exponents for each parameter, $\langle\chi\rangle$ has the following dependence:

$$
\langle\chi\rangle=\left(C_{1} f_{\mathrm{ren}} n^{\alpha_{n}} P^{\alpha_{P}} B^{\alpha_{B}} a^{\alpha_{a}-2} R^{\alpha_{R}}\right)^{-1},
$$

where $C_{1}$ is a constant. From the relation of $\tau_{\mathrm{E}} \propto n T V / P$, where $T$ is the temperature, and $V \sim 2 \pi^{2} a^{2} R$ is the volume,

$$
P \propto n T V\langle\chi\rangle / a^{2}=2 \pi^{2} n T R\langle\chi\rangle
$$




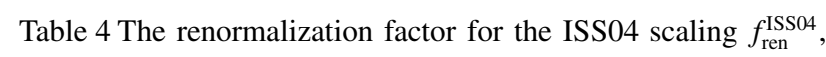
the renormalization factor for the local transport coefficients $g_{\mathrm{ren} \chi}^{\mathrm{IS} 04}$, and the geometric center position of the $\rho=2 / 3$ magnetic flux surface $R_{\mathrm{geo}}^{\mathrm{vac}}(2 / 3)$, in three magnetic configurations on LHD $\left(R_{\mathrm{ax}}^{\mathrm{vac}}=3.60,3.75\right.$, and $3.90 \mathrm{~m})$.

\begin{tabular}{|l|l|l|l|}
\hline$R_{\mathrm{ax}}^{\mathrm{Vac}}[\mathrm{m}]$ & $R_{\mathrm{geo}}^{\mathrm{vac}}(2 / 3)[\mathrm{m}]$ & $f_{\mathrm{ren}}^{\mathrm{ISS} 04}$ & $g_{\mathrm{ren} \chi}^{\mathrm{ISS} 04}$ \\
\hline 3.60 & 3.643 & 0.93 & 1.00 \\
\hline 3.75 & 3.740 & 0.67 & 2.33 \\
\hline 3.90 & 3.849 & 0.48 & 5.48 \\
\hline
\end{tabular}

is derived. Then, a scaling for $\langle\chi\rangle$ is expressed as

$$
\begin{aligned}
\langle\chi\rangle= & \left(\langle\chi\rangle^{\alpha_{P}} C_{2} f_{\mathrm{ren}} n^{\alpha_{n}+\alpha_{P}} B^{\alpha_{B}} a^{\alpha_{a}-2} R^{\alpha_{R}+\alpha_{P}} T^{\alpha_{P}}\right)^{-1} \\
= & \left(C_{2} f_{\mathrm{ren}} n^{\alpha_{n}+\alpha_{P}} B^{\alpha_{B}} a^{\alpha_{a}-2} R^{\alpha_{R}+\alpha_{P}} T^{\alpha_{P}}\right)^{-1 /\left(\alpha_{P}+1\right)} \\
= & f_{\mathrm{ren}}^{-1 /\left(\alpha_{P}+1\right)} C_{2}^{-1 /\left(\alpha_{P}+1\right)} \\
& \times\left(n^{\alpha_{n}+\alpha_{P}} B^{\alpha_{B}} a^{\alpha_{a}-2} R^{\alpha_{R}+\alpha_{P}} T^{\alpha_{P}}\right)^{-1 /\left(\alpha_{P}+1\right)}
\end{aligned}
$$

where $C_{2}$ is another constant. The local renormalization factor based on the global scaling ISS04, $g_{\mathrm{ren} \chi}^{\mathrm{ISS} 04}$ is derived by normalizing $f_{\text {ren }}^{-1 /\left(\alpha_{P}+1\right)}$ with the value at $R_{\mathrm{ax}}^{\mathrm{vac}}=3.60 \mathrm{~m}$ as the following,

$$
g_{\mathrm{ren} \chi}^{\mathrm{ISS} 04} \equiv f_{\mathrm{ren}}^{-1 /\left(\alpha_{P}+1\right)} / f_{\mathrm{ren}}^{-1 /\left(\alpha_{P}+1\right)}\left(R_{\mathrm{ax}}^{\mathrm{vac}}=3.6 \mathrm{~m}\right)
$$

where $\alpha_{P}=-0.61$. The values of $g_{\mathrm{ren} \chi}^{\mathrm{ISS} 04}$ at the $R_{\mathrm{ax}}^{\mathrm{vac}}=$ $3.60,3.75$, and $3.90 \mathrm{~m}$ configurations are shown in Table 4 .

It should be noted that $\langle\chi\rangle$ in the $R_{\mathrm{ax}}^{\mathrm{vac}}=3.90 \mathrm{~m}$ configuration is about five times larger than that in the $R_{\mathrm{ax}}^{\mathrm{vac}}=$ $3.60 \mathrm{~m}$ configuration when its evaluation is based on ISS04. The values of $g_{\text {ren } \chi}^{\text {ISS04 }}$ represent the predicted change in the mean local transport coefficients, when the confinement time changes by the factor of $f_{\mathrm{ren}}^{\mathrm{ISS} 04} / f_{\mathrm{ren}}^{\mathrm{ISS} 04}\left(R_{\mathrm{ax}}^{\mathrm{vac}}=\right.$ $3.6 \mathrm{~m})$. They almost agree with the values of $g_{\text {ren } \chi}$, which are evaluated from the local transport analysis from the results in Fig. 10. Here, the $\rho=2 / 3$ surface is selected as the flux surface corresponding to $g_{\mathrm{ren} \chi}^{\text {ISS04 }}$, because it is assumed that $R_{\text {geo }}(2 / 3)$ represents the property of a magnetic configuration.

\section{Local Transport Property in the High-Beta Regime, and its Depen- dence on the Magnetic Configura- tion}

In Sec. 3, it is suggested that the degradation of the global confinement property may be explained by the change in the magnetic flux surface, which corresponds to the torus-outward shift of the geometric center of the $\rho=2 / 3$ magnetic flux surface, due to the increment in beta. On the other hand, it is not clear that the same explanation can be valid from the viewpoint of the local trans- (a)

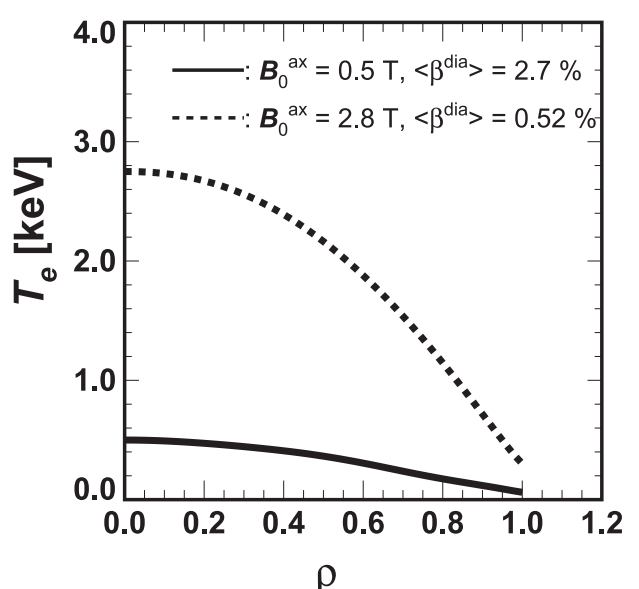

(b)

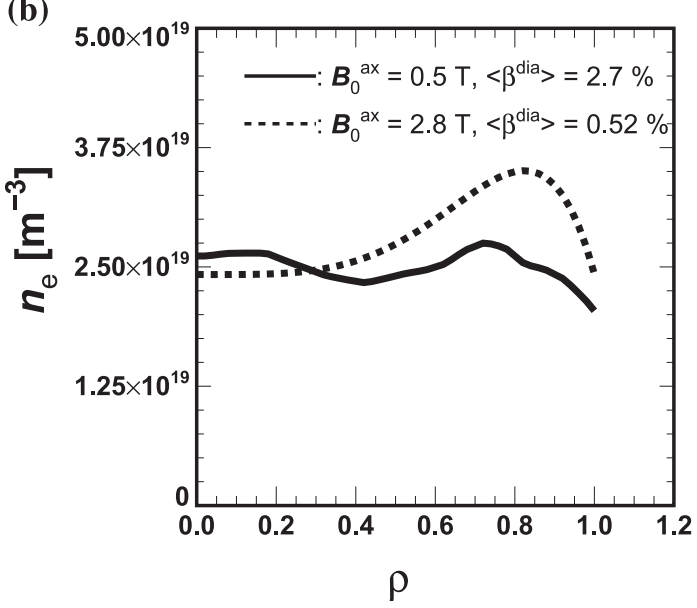

Fig. 11 Examples of modeled (a) $T_{\mathrm{e}}$ and (b) $n_{\mathrm{e}}$ profiles (solid curves: $B_{0}^{\text {ax }}=0.5 \mathrm{~T},\left\langle\beta^{\text {dia }}\right\rangle=2.7 \%$ and broken curves: $\left.B_{0}^{\mathrm{ax}}=2.8 \mathrm{~T},\left\langle\beta^{\mathrm{dia}}\right\rangle=0.52 \%\right)$.

port. In this section, the behavior of local transport coefficients in high-beta plasmas is studied by referencing $\chi^{\text {ISS04 }}$.

Two typical examples of $T_{\mathrm{e}}$ and $n_{\mathrm{e}}$ profiles in the LHD experiments are shown in Fig. 11 (a) and (b), respectively. These profiles are derived by fitting the experimental data with certain functions. The solid curves represent the plasma produced in $B_{0}^{\mathrm{ax}}=0.5 \mathrm{~T}$ and with $\left\langle\beta^{\text {dia }}\right\rangle=2.7 \%$. The broken curves represent the plasma produced in $B_{0}^{\mathrm{ax}}=2.8 \mathrm{~T}$ with $\left\langle\beta^{\text {dia }}\right\rangle=0.52 \%$. These plasmas were obtained with the same preset magnetic axis position, which corresponds to the magnetic axis position in vacuum $R_{\mathrm{ax}}^{\mathrm{vac}}$. Examples of profiles of $\chi^{\mathrm{eff}} / \chi_{\mathrm{e}}^{\mathrm{GRB}}$ are shown in Fig. 12, where the transport coefficient by the GRB model, $\chi^{\mathrm{GRB}}=T_{\mathrm{e}} / e B \cdot \rho_{\mathrm{s}} / a$, and $\rho_{\mathrm{s}}$ is the ion gyroradius measured using $T_{\mathrm{e}}$. Here, the values of $B_{0}^{\text {ax }}$ are used for $B$. The coefficient $\chi^{\mathrm{GRB}}$ is used since $\chi^{\mathrm{ISS} 04}$ includes a factor $C_{\chi}$, which has different values depending on the $\rho$ positions. The distinction of the curves is the same in Fig. 11. Although the magnitude of $\chi^{\text {eff }}$ differs between $B_{0}^{\mathrm{ax}}=0.5$ and $2.8 \mathrm{~T}$, the ratio $\chi^{\mathrm{eff}} / \chi_{\mathrm{e}}^{\mathrm{GRB}}$ has a similar mag- 


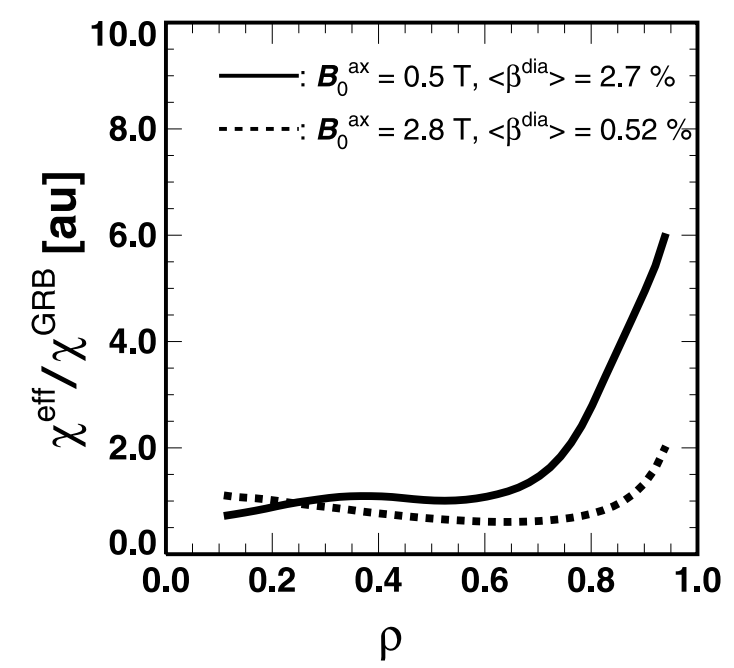

Fig. 12 Examples of experimentally derived $\chi^{\mathrm{eff}} / \chi_{\mathrm{e}}^{\mathrm{GRB}}$ profiles (solid curve: $B_{0}^{\mathrm{ax}}=0.5 \mathrm{~T},\left\langle\beta^{\mathrm{dia}}\right\rangle=2.7 \%$ and broken curve: $\left.B_{0}^{\mathrm{ax}}=2.8 \mathrm{~T},\left\langle\beta^{\text {dia }}\right\rangle=0.52 \%\right)$.

nitude in the high-beta and low-beta cases except at the peripheral region of $\rho>0.7$.

Figure $13(\mathrm{a}),(\mathrm{b})$, and (c) shows the dependence of $\chi^{\text {eff }} / \chi^{\mathrm{ISS} 04}$ on $\left\langle\beta^{\text {dia }}\right\rangle$ of the $R_{\mathrm{ax}}^{\mathrm{vac}}=3.6 \mathrm{~m}$ plasmas at $\rho=$ $0.5,0.7$, and 0.9 , respectively. Degradation of $\chi^{\mathrm{eff}} / \chi^{\mathrm{ISS} 04}$ is observed in the $\left\langle\beta^{\text {dia }}\right\rangle>1 \%$ region at all these minor radial positions. To distinguish the direct effects of $\beta$ from those in the change in the magnetic configuration by the increment in beta as the causes of this degradation, the dependence of $\chi^{\mathrm{eff}} / \chi^{\mathrm{ISS} 04}$ on the geometric center position of the magnetic flux surface $R_{\text {geo }}(\rho)$ in high-beta plasmas is studied. The results is compared with the dependence of $\chi^{\mathrm{eff}} / \chi^{\mathrm{ISS} 04}$ on the magnetic configuration in the low-beta region, which is evaluated from the results in different $R_{\mathrm{ax}}^{\mathrm{vac}}$ plasmas in the previous section.

From the relation between $R_{\text {geo }}(\rho)$ and $\left\langle\beta^{\text {dia }}\right\rangle$ at $\rho=$ $0.5,0.7$, and 0.9 , the outside shift of the geometric center of the magnetic flux surface becomes large in the $\left\langle\beta^{\text {dia }}\right\rangle>1 \%$ region at all these minor radial positions. It is found that the magnitude of the shift is larger in the core region than that in the peripheral region.

In Fig. 14, the dependence of $\chi^{\text {eff }} / \chi^{\mathrm{ISS} 04}$ on $R_{\text {geo }}(\rho)$ in the high-beta regime is compared with the dependence of $\chi^{\text {eff }} / \chi^{\text {ISS04 }}$ on $R_{\text {geo }}(\rho)$ in the low-beta regime with various magnetic axis positions. Figures 14 (a), (b), and (c) show the results for $\rho=0.5,0.7$, and 0.9 , respectively. The closed circles $(\bullet)$ represent the relation between $\chi^{\text {eff }} / \chi^{\text {ISS04 }}$ and $R_{\text {geo }}(\rho)$. Although $R_{\text {geo }}(\rho)$ depends on the pressure profile of the plasma, the data at the larger $R_{\text {geo }}(\rho)$ position have higher $\beta$ values in general. These experimental data are same as used in Fig. 13. At all positions of $\rho=0.5,0.7$, and 0.9 , the magnitude of $\chi^{\text {eff }} / \chi^{\mathrm{ISS} 04}$ increases with the torus-outward shift of $R_{\text {geo }}(\rho)$ of the each magnetic flux surface. The dependence of the increment in the ratio $\chi^{\text {eff }} / \chi^{\mathrm{ISS} 04}$ on $R_{\text {geo }}(\rho)$ is steeper at the larger $\rho$ posi-

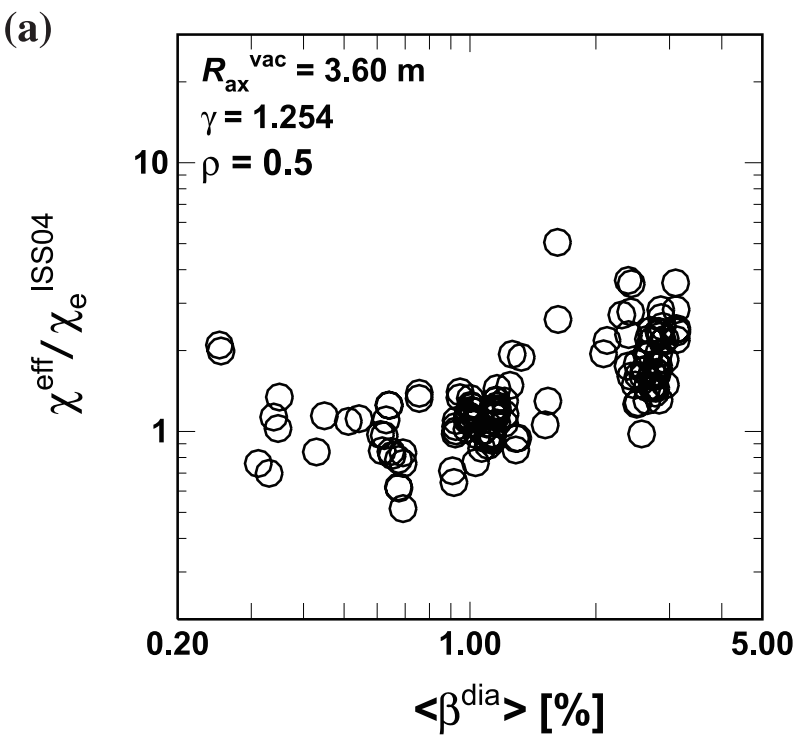

(b)

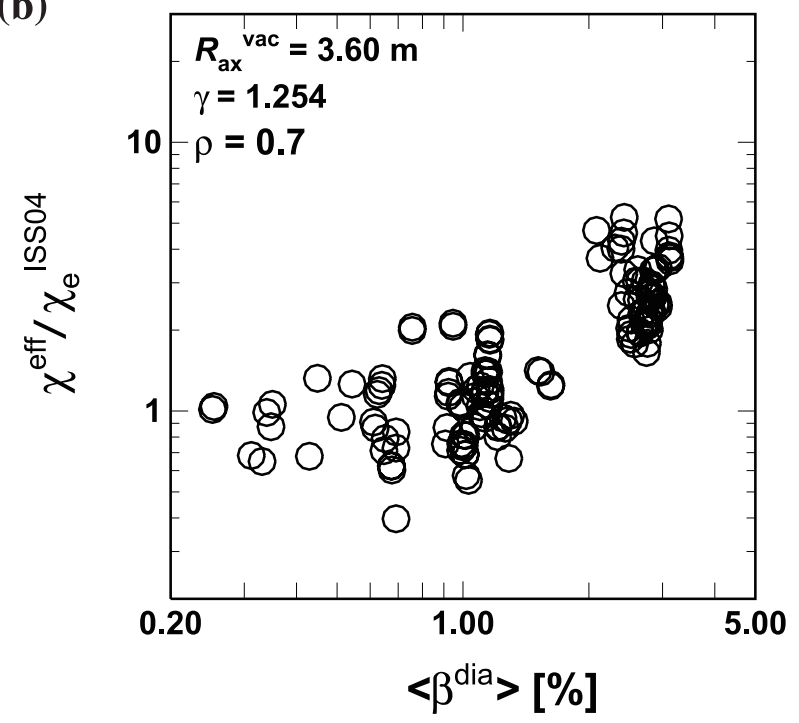

(c)

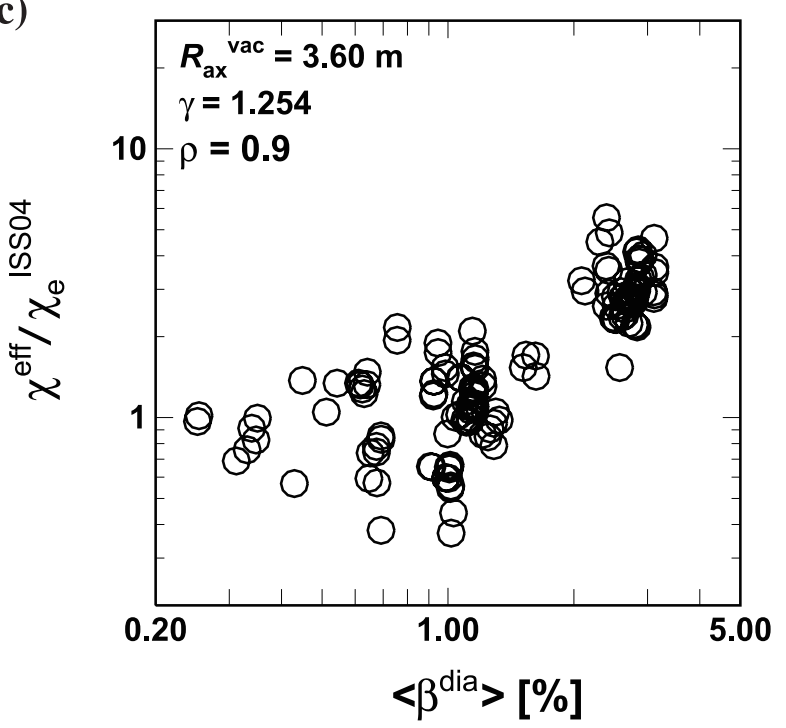

Fig. 13 Dependence of $\chi^{\text {eff }} / \chi^{\text {ISS04 }}$ on $\left\langle\beta^{\text {dia }}\right\rangle$ in the magnetic configuration of $R_{\mathrm{ax}}^{\mathrm{vac}}=3.60 \mathrm{~m}$. (a) $\rho=0.5$ (b) $\rho=0.7$, and (c) $\rho=0.9$. 
(a)

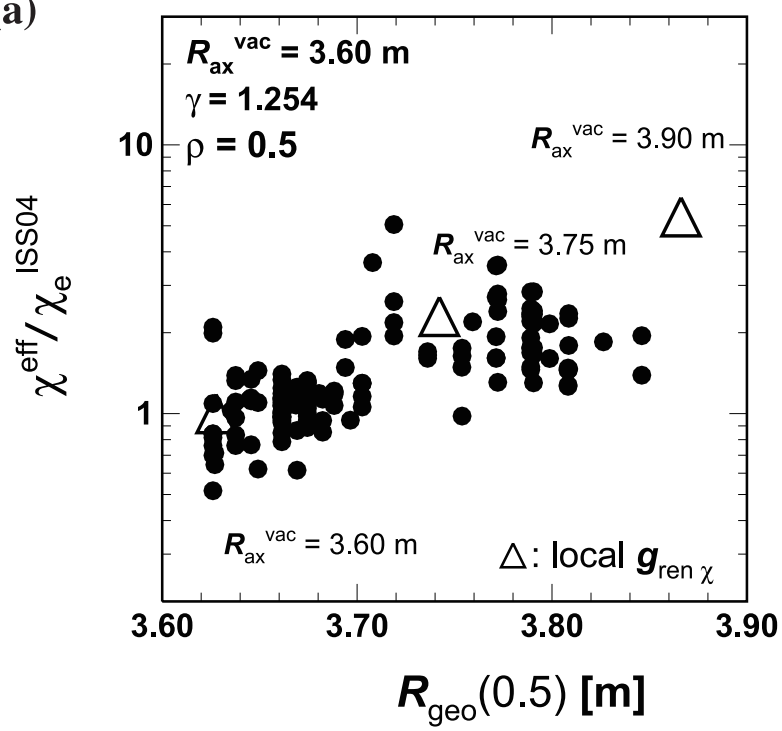

(b)

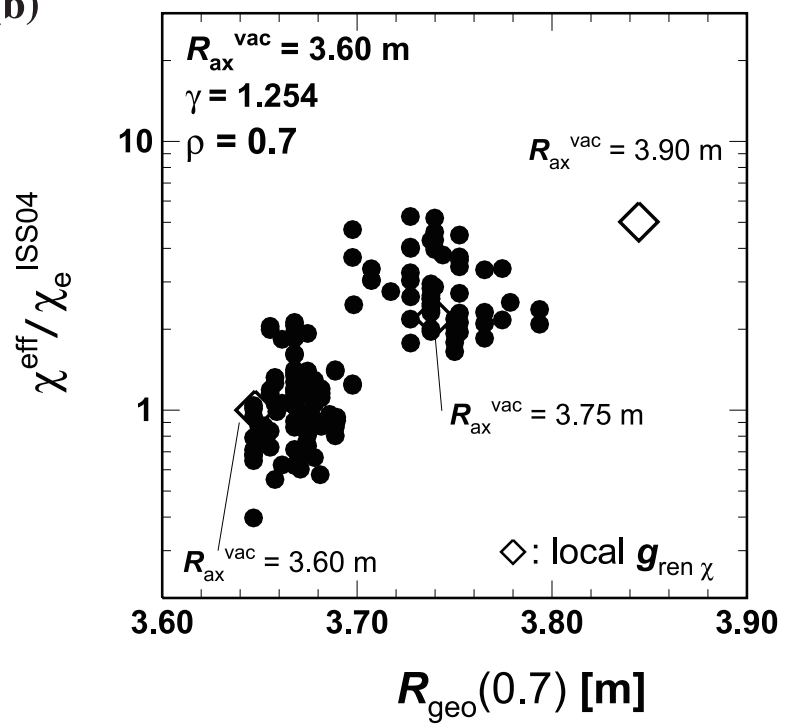

(c)

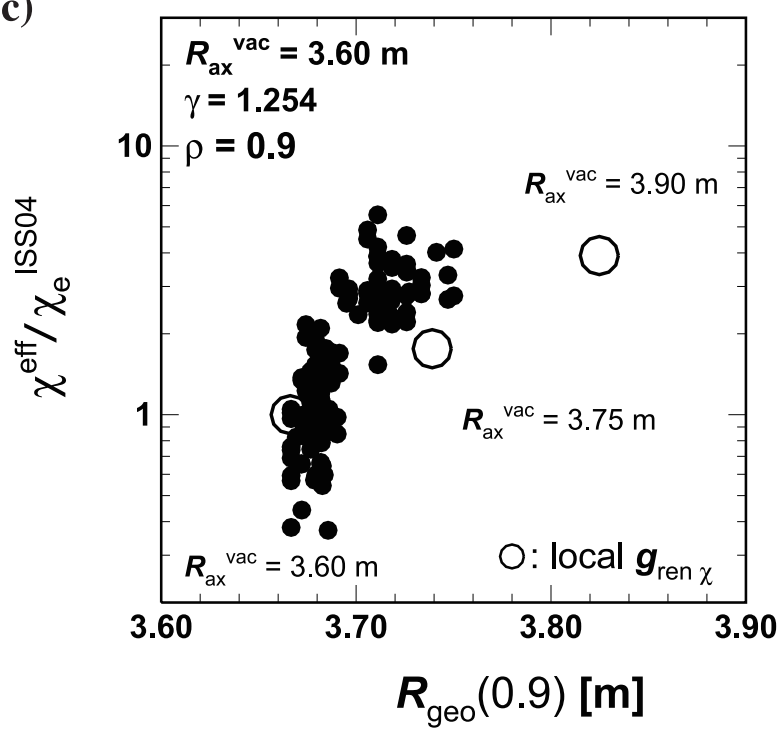

Fig. 14 Dependence of the local transport coefficients $\chi^{\text {eff }} / \chi^{\text {ISS04 }}$ on $R_{\text {geo }}(\rho)$. (a) $\rho=0.5$, (b) $\rho=0.7$, and (c) $\rho=0.9$. tion. One reason of observed is that the magnitude of the shift of $R_{\text {geo }}(\rho)$ is smaller at larger $\rho$. At $\rho=0.9$, an abrupt increment in $\chi^{\mathrm{eff}} / \chi^{\mathrm{ISS} 04}$ is found around $R_{\mathrm{geo}}(\rho) \simeq 3.70 \mathrm{~m}$, where $\left\langle\beta^{\text {dia }}\right\rangle$ is about $1.0 \sim 2.5 \%$. The symbols $\Delta, \diamond$, and $\bigcirc$ in Fig. 14 are the same as in Fig. 10. They represent the dependence of the normalized thermal transport coefficients at the geometric center position of the magnetic flux surfaces which is evaluated from the local transport analysis.

The degradation of the local transport with the increment in $\left\langle\beta^{\text {dia }}\right\rangle$ seems to be comparable with the degradation by the torus-outward shift of the magnetic flux surface at $\rho=0.7$. Moreover, the degradation at $\rho=0.5$ seems to be comparable with or slightly smaller than the degradation by the torus-outward shift. On the other hand, particularly at the peripheral region of $\rho=0.9$ as shown in Fig. 14 (c), it is observed that the degradation of the local transport is larger than that predicted from the torus-outward shift of the magnetic flux surface. This results shows that some effects caused directly by beta may exist in the peripheral region.

\section{Discussion and Summary}

The following results are obtained by comparing the dependence of the local transport coefficients on beta values and the geometric center positions of the magnetic flux surfaces with the relations between the local transport coefficients and the geometric center positions of the magnetic flux surfaces in the low-beta region. The degradation of the local transport with the increment in $\langle\beta\rangle$ seems to be comparable with or is slightly smaller than the degradation by the torus-outward shift of the magnetic flux surface at $\rho=0.7$ or 0.5 . On the other hand, at the peripheral region of $\rho=0.9$, degradation of transport coefficients larger than that predicted from the torus-outward shift is observed. It is considered that some effects that are directly caused by the beta value or the gradient of beta may exist.

From the results of the local transport analysis, although the degradation due to $\langle\beta\rangle$ seems to be explained by the change in the magnetic configuration from the global confinement analysis, local transport degradation by some effects directly caused by the increment in beta other than the change in the magnetic configuration may exist in the peripheral region. One possible explanation for this difference is as follows. In the peripheral region near $\rho=0.9$, effects other than the change in the magnetic configuration may dominate. On the other hand, in the inner region near $\rho=0.5$, the transport degradation directly due to beta is smaller than that by the configuration effect. The degradation of global confinement property by $\langle\beta\rangle$ seems to be similar to the configuration effect, since these effects are balanced. As one of the causes of the transport degradation in the peripheral region, the resistive g-mode turbulence is considered, since the heliotron-type magnetic configuration has a magnetic hill at the peripheral region. Analysis 
of this effect is shown in Ref. [5]. Although the effect of increment in ergodicity at the edge exists as another candidate for the causes of the transport degradation, it is shown that well-closed magnetic flux surfaces are still found at $\rho=0.9$ from the calculation results of HINT2 [12], which is a 3-dimensional MHD equilibrium code [13].

\section{Acknowledgements}

The authors would like to thank Profs. O. Motojima, S. Sudo and A. Komori for their continuous encouragement. This study has been supported at NIFS by NIFS07ULHH519.

[1] O. Motojima et al., Proc. 21st IAEA Fusion Energy Conf., OV/2-1, (Chengdu, 2006).

[2] U. Stroth, M. Murakami et al., Nucl. Fusion 36, 1063
(1996).

[3] H. Yamada et al., Nucl. Fusion 45, 1684 (2005).

[4] J. Miyazawa et al., Plasma Phys. Control. Fusion 47, 801 (2005).

[5] H. Funaba, K.Y. Watanabe et al., Fusion Sci. Tech. 51, 129 (2007).

[6] B.A. Carreras and P.H. Diamond, Phys. Fluids B 1, 1011 (1989).

[7] K.Y. Watanabe et al., Nucl. Fusion 45, 1247 (2005).

[8] S. Murakami et al., Trans. Fusion Technol. 27, 256 (1995).

[9] M. Osakabe, Y. Takeiri et.al., Rev. Sci. Instrum. 72, 590 (2001).

[10] H.C. Howe, ORNL/TM-11521 (1990).

[11] K.Y. Watanabe et al., Plasma Phys. Control. Fusion 49, 605 (2007).

[12] Y. Suzuki et al., Proc. 17th Intern. Toki Conf. (Toki, 2007) (to be published).

[13] K. Harafuji, T. Hayashi et al., J. Comput. Phys. 81, 169 (1989). 